\title{
Mode coupling enhancement from dielectric to plasmonic waveguides
}

\author{
Rami A. Wahsheh $\odot^{*}$ \\ Princess Sumaya University for Technology, King Abdullah II School of Engineering, \\ Communications Engineering Department, Al-Jubaiha, Amman, Jordan
}

\begin{abstract}
Efficient dielectric-plasmonic interconnect is significant in the design of future electronic-photonic integrated circuits that deal with large data transfer. We propose three designs and analysis of small-footprint couplers that are located at the interface between dielectric and plasmonic waveguides. To the best of our knowledge, our proposed couplers outperform all the works reported in the literature in several aspects including size and coupling efficiency (CE). Our results indicate that the optimum dimensions of the proposed couplers are determined based on whether the coupler is located in the metal side or dielectric side, or the coupler extends equally in both types of materials. The proposed couplers work over a broad frequency range achieving a CE above $88 \%$ at the optical communications wavelength $1550 \mathrm{~nm}$. In addition, our results indicate that the CE can be further increased to above $93 \%$ by increasing the width of the dielectric waveguide before it is connected to the coupler. Moreover, our proposed designs provide a considerable alignment tolerance, which is needed when aligning the dielectric waveguide to the metal-dielectric-metal waveguide. Our proposed couplers have an impact on the design and miniaturization of nanoscale all-optical devices. () The Authors. Published by SPIE under a Creative Commons Attribution 4.0 Unported License. Distribution or reproduction of this work in whole or in part requires full attribution of the original publication, including its DOI. [DOI: 10.1117/1.OE.59.10 $.107101]$
\end{abstract}

Keywords: integrated optics devices; waveguides; surface plasmons.

Paper 20200851 received Jul. 13, 2020; accepted for publication Sep. 23, 2020; published online Oct. 8, 2020.

\section{Introduction}

Metal-dielectric-metal (MDM) waveguides confine light in a subwavelength-sized dielectric region, which is needed for the future of on-chip electronic-photonic integrated circuits. ${ }^{1,2}$ The propagation losses in the MDM waveguides are high due to their large metallic losses, which have prevented using them to propagate light over long distances. On the other hand, the conventional dielectric waveguides have very low propagation losses and are used to propagate light over long distances. To use the MDM waveguides in the fabrication of plasmonic devices, such as splitters, ${ }^{3}$ Mach-Zehnder interferometers, ${ }^{4}$ reflectors, ${ }^{5}$ wavelength demultiplexers, ${ }^{6}$ circulators, ${ }^{7}$ filters, ${ }^{8}$ and all-optical switching, ${ }^{9}$ integrating both dielectric and plasmonic waveguides on the same chip is needed. Consequently, an efficient coupler between those two kinds of waveguides is needed, in which the dielectric waveguides are used for transmission of light into and out of the MDM waveguides. Several different coupling methods have been proposed to achieve mode matching between those two types of waveguides: some designed a coupler and placed it inside the metal side, ${ }^{3,10,11}$ others placed it inside the dielectric side, ${ }^{12,13}$ and some others placed it inside both sides. ${ }^{14-16}$ Aligning the dielectric waveguide to the MDM waveguide is challenging as demonstrated in Refs. 12 and 17-19, in which considerable alignment tolerance is needed.

In this research work, we propose three designs of ultracompact couplers to increase the coupling efficiency (CE) from a 300-nm-wide silicon waveguide into a 40-nm silver-air-silver plasmonic waveguide. In our previous work, ${ }^{3}$ we achieved a CE of $88 \%$ by incorporating an ultrashort rectangular air-gap at the interface between dielectric and MDM plasmonic

*Address all correspondence to Rami A. Wahsheh, E-mail: r.wahsheh@psut.edu.jo 


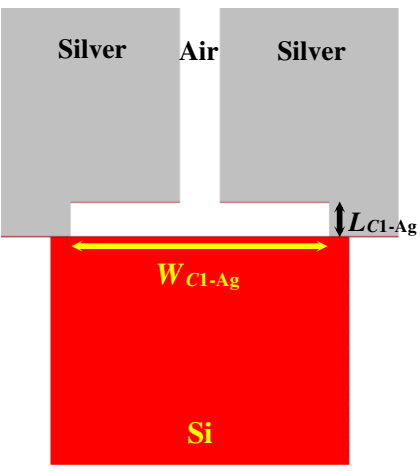

(a)

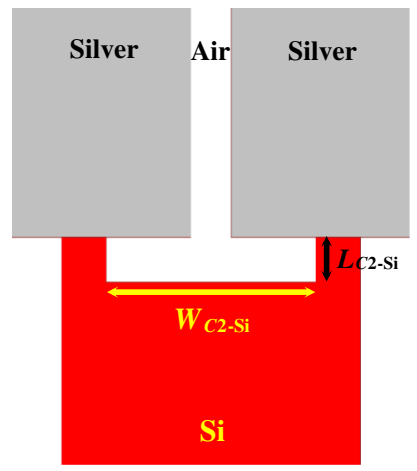

(b)

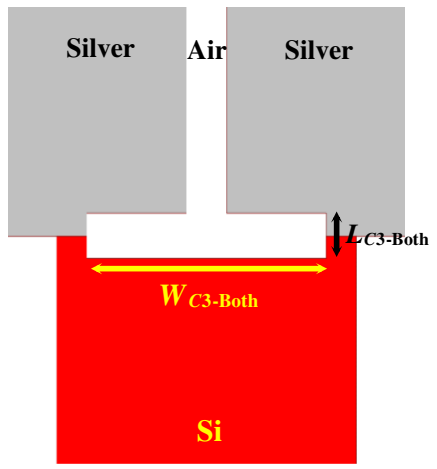

(c)

Fig. 1 Schematics of the proposed three AGCs: (a) AGC \#1 inside the metal region, (b) AGC \#2 inside the dielectric region, and (c) AGC \#3 inside both materials.

waveguides. The width of the air-gap-coupler (AGC) matched that of the dielectric waveguide and was located inside the metal region. Herein, we show a different approach other than that followed in our previous work to design three types of couplers. Our proposed three AGCs are all located at the interface between the dielectric and MDM plasmonic waveguides: AGC \#1 is located inside the metal region [as shown in Fig. 1(a)], AGC \#2 is located inside the dielectric region [as shown in Fig. 1(b)], and AGC \#3 extends equally inside both types of materials [as shown in Fig. 1(c)].

The proposed couplers were designed using the two-dimensional finite-difference timedomain method with a uniform mesh size of $1 \mathrm{~nm}$ to accurately capture the field changes at the interface between the dielectric and MDM waveguides. In all simulations, the fundamental mode was excited in the dielectric waveguide, and the $\mathrm{CE}$ was measured close to the interface with the plasmonic waveguide. The $\mathrm{CE}$ is defined as the ratio of the transmitted light power into the MDM waveguide to that launched into the dielectric waveguide. All numerical simulations were done at the optical communications wavelength $1550 \mathrm{~nm}$ taking into account the propagation losses in the MDM waveguides.

Herein, we present the design steps and numerical results for our proposed AGCs. The remainder of this research work is organized as follows. In Sec. 2, we explain the design procedures and results. Also, we show how the proposed designs increase the fabrication tolerance, which is needed when aligning the dielectric waveguide to the plasmonic waveguide. In Sec. 3, we compare the spectrum results of each proposed design. In Sec. 4, we provide a conclusion.

\section{Air-Gap-Coupler Design and Analysis}

Our proposed AGCs are all located at the interface between the dielectric and MDM plasmonic waveguides. In the simulations, the dimensions of the couplers (i.e., length and width) were changed and the corresponding CE was measured at $10 \mathrm{~nm}$ away from the AGC boundary. The length of the coupler, $L_{C}$, was changed in steps of $5 \mathrm{~nm}$ from 5 to $50 \mathrm{~nm}$. For each step of $L_{C}$, the width of the coupler, $W_{C}$, was changed from 0 to $400 \mathrm{~nm}$ (as shown in Fig. 2), where 0 means direct connection of the two types of waveguides. We found that the CE for AGC \#1 was above $89 \%$ that occurred at $L_{C 1-\mathrm{Ag}}=15 \mathrm{~nm}$ and $W_{C 1-\mathrm{Ag}}=260 \mathrm{~nm}$ [as shown in Fig. 2(a)]. Whereas the CE for AGC \#2 was about $88 \%$ that occurred at $L_{C 2-\mathrm{Si}}=20 \mathrm{~nm}$ and $W_{C 2-\mathrm{Si}}=$ $210 \mathrm{~nm}$ [as shown in Fig. 2(b)] and that for AGC \#3 was about $89 \%$ that occurred at $L_{C 3-\text { Both }}=$ $20 \mathrm{~nm}$ and $W_{C 3-\text { Both }}=240 \mathrm{~nm}$ [as shown in Fig. 2(c)]. Light couples into the AGC before it is funneled into the MDM waveguide. Without using the AGC, the CE was about $68 \%$ in which there was a large mode-size mismatch between the size of the mode in the dielectric waveguide and that in the MDM waveguide. In our proposed designs, using the AGC increased the CE to 


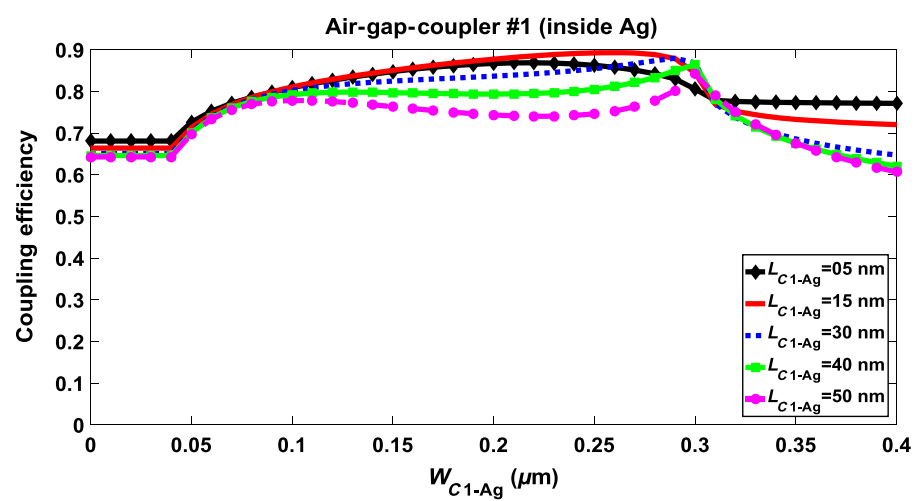

(a)

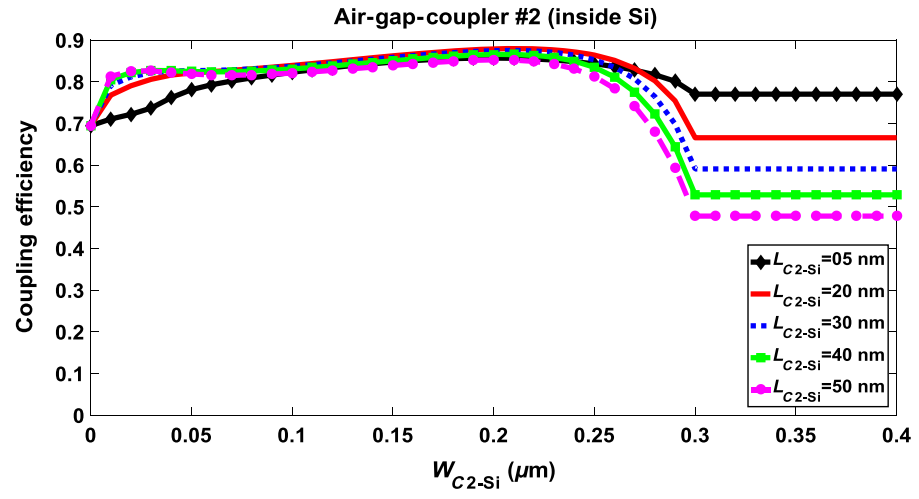

(b)

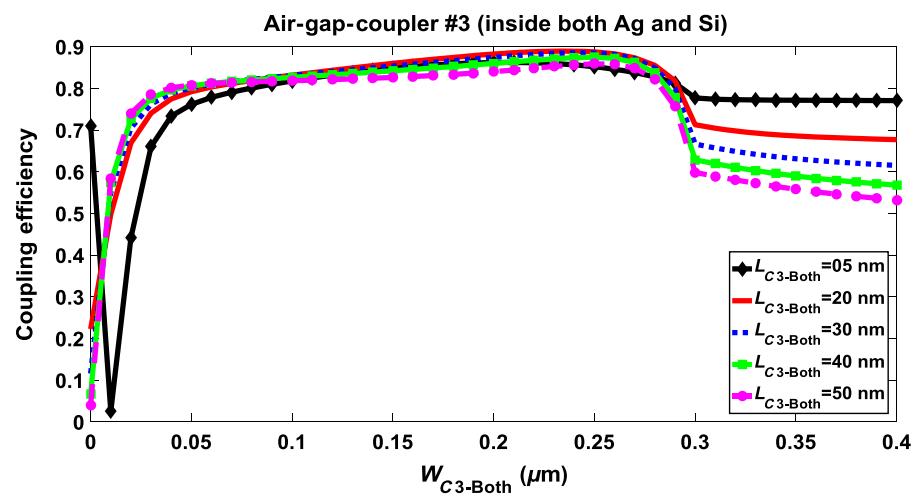

(c)

Fig. $2 \mathrm{CE}$ as a function of the width of the AGC, $W_{C}$, for different lengths of the coupler, $L_{C}$, applied for all the proposed designs of couplers: (a) AGC \#1, (b) AGC \#2, and (c) AGC \#3.

above $88 \%$ by reducing the mode-size mismatch between the two waveguides and by reducing the transverse leakage at the interface between the dielectric and MDM waveguides.

Moreover, we found that the $\mathrm{CE}$ of all three proposed designs that are shown in Fig. 1 can be further increased by gradual increase of the width of the dielectric waveguide, $W_{\mathrm{Si}}$, over a length $L_{\mathrm{Si}}$ before it is connected to the AGC (see Fig. 3). In the simulations, the corresponding CE was measured at $10 \mathrm{~nm}$ away from the AGC boundary taking into account the propagation losses in the tapered-dielectric waveguides. We found that, for AGC \#1, the CE increased from $89 \%$ to above $93 \%$ when the width of the dielectric waveguide was expanded to $W_{\mathrm{Si}-C 1}=380 \mathrm{~nm}$ over a length of $L_{\mathrm{Si}-C 1}=330 \mathrm{~nm}$ [as shown in Fig. 4(a)]. Whereas for AGC \#2, the CE increased from $88 \%$ to above $93 \%$ when the width of the dielectric waveguide was expanded to $W_{\mathrm{Si}-C 2}=$ $380 \mathrm{~nm}$ over a length of $L_{\mathrm{Si}-C 2}=340 \mathrm{~nm}$ [as shown in Fig. 4(b)], and for AGC \#3, the CE 


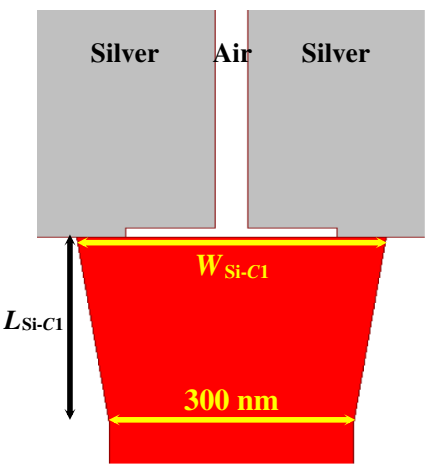

(a)

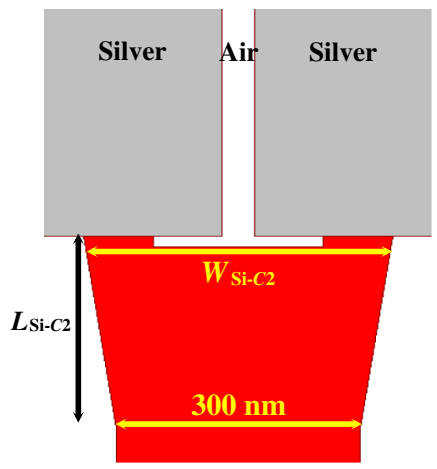

(b)

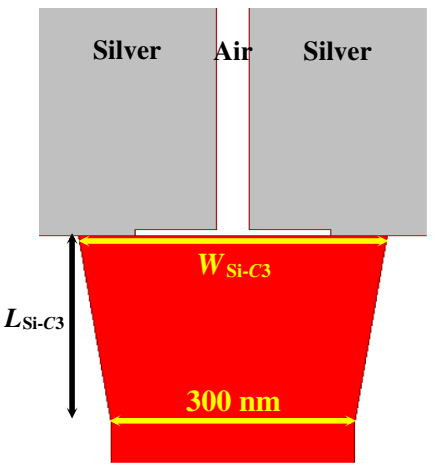

(c)

Fig. 3 Schematics of the proposed designs for the AGCs after gradual increase of the width of the dielectric waveguide, $W_{\mathrm{Si}}$, over a length, $L_{\mathrm{Si}}$, for: (a) AGC \#1, (b) AGC \#2, and (c) AGC \#3.

increased from $89 \%$ to above $93 \%$ when the width of the dielectric waveguide was expanded to $W_{\mathrm{Si}-C 3}=380 \mathrm{~nm}$ over a length of $L_{\mathrm{Si}-C 3}=330 \mathrm{~nm}$ [as shown in Fig. 4(c)]. The gradual increase of the width of the dielectric waveguide acted as a fine-tuning-mode matching that enabled more light to couple into the AGC and consequently resulted in increasing the $\mathrm{CE}$ into the MDM waveguide.

We also found that our proposed couplers provide a considerable fabrication tolerance when aligning the dielectric waveguide to the MDM waveguide. To show that, the misalignment between the center of the dielectric waveguide and that of the MDM waveguide was changed as a function of the misalignment distance, $d$ (see Fig. 5). In the simulations, the AGC was kept connected to the MDM waveguide for all designs as if the focused ion beam (FIB) was used to define both the MDM waveguide and AGC. Figure 6 shows the CE as a function of the misalignment distance $d$ for all the proposed AGCs. We increased $d$ until there was no overlap between the two waveguides. We found that the CE decreased for all types of couplers as $d$ was increased. For example, when $d=100 \mathrm{~nm}$, the CE was above $40 \%$ for all proposed couplers compared to about $8 \%$ without using the AGC (i.e., butt coupling). From the results shown in Fig. 6, the misalignment tolerance was much higher when using the AGC and maximum CE occurs when the center of the AGC was aligned to that of the dielectric waveguide.

\section{Spectrum Analysis}

All our proposed AGCs operate at a broad frequency range. To show that, we varied the wavelength of the light source from 900 to $2300 \mathrm{~nm}$ and measured the corresponding CE at each wavelength. The spectrum of the proposed couplers that are shown in Figs. 1 and 3 are all shown in Fig. 7. For comparison, we also added the spectrum of the butt coupling of the two waveguides. Using the AGCs broadens the spectrum range around the communications wavelength of $1550 \mathrm{~nm}$ for all the proposed couplers compared to that without using the AGC. As shown in Fig. 7, the spectrum of AGC \#2 without $\mathrm{Si}$ width expansion has better $\mathrm{CE}$ than the other couplers when the wavelength is $<1370 \mathrm{~nm}$, whereas the spectrum of AGC \#1 with Si width expansion has better CE than the other couplers when the wavelength is $>1370 \mathrm{~nm}$. The coupler that has very close results to the best coupler before and after the $1370 \mathrm{~nm}$ is AGC \#3 that extends equally in both types of waveguides. Based on these results and since we do not have high precision fabrication techniques, using AGC \#3 is our best choice especially when using the FIB to define both the AGC and the MDM waveguides. It is almost impossible to fabricate AGC \#1 inside the metal region that exactly starts at the interface with the dielectric waveguide without having part of the coupler in the dielectric waveguide. The same scenario can be said about fabricating AGC \#2. Our only choice is to fabricate AGC \#3 that extends into both types of waveguides. Figure 2(c) shows that the $\mathrm{CE}$ is $>50 \%$ when the coupler's width, $W_{C 3-B o t h}$ is between 30 and 


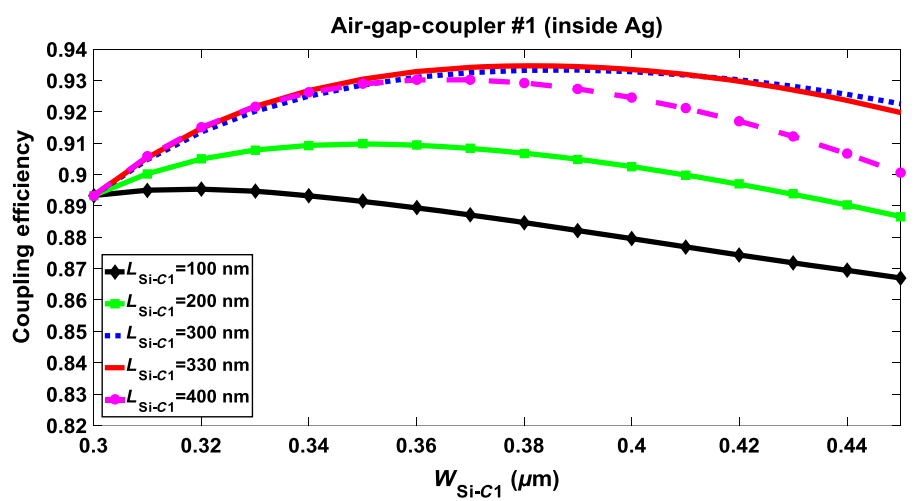

(a)

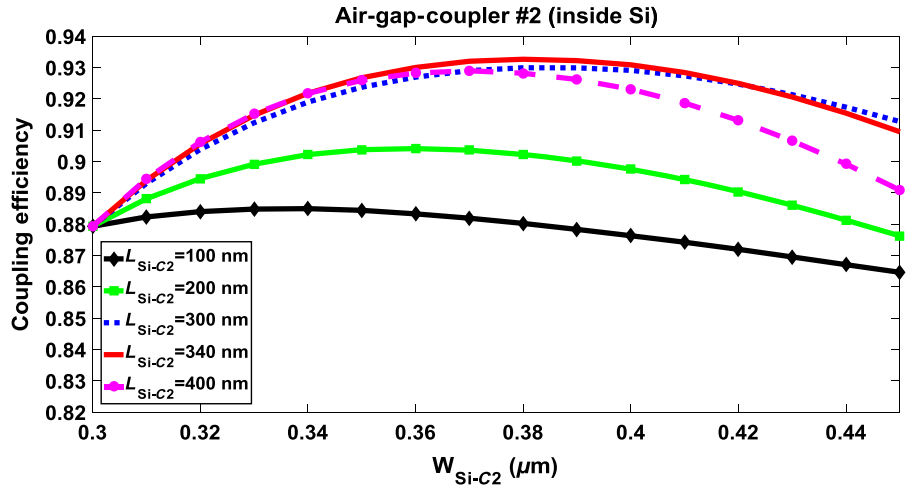

(b)

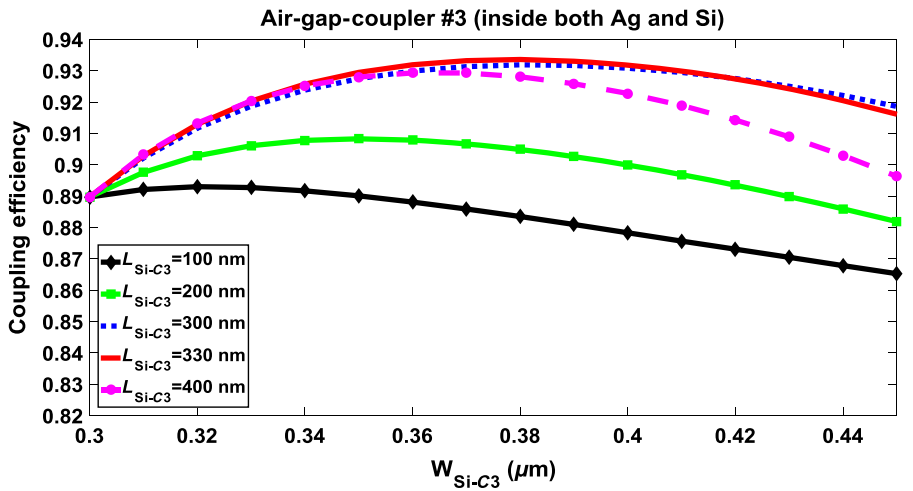

(c)

Fig. $4 \mathrm{CE}$ of the optimum designs of the AGCs as a function of the dielectric waveguide width, $W_{\mathrm{Si}}$, that increased gradually over different lengths, $L_{\mathrm{Si}}$, for (a) AGC \#1, (b) AGC \#2, and (c) AGC \#3.

$400 \mathrm{~nm}$ for any coupler's length, $L_{C 3-B o t h}$ is between 5 and $50 \mathrm{~nm}$. This means that as long as there is an overlap between AGC \#3 and the two types of waveguides, light couples from the dielectric waveguide into the plasmonic waveguide, which can be achieved using the FIB. To fabricate AGC \#3 on a 250-nm silicon-on-insulator wafer, the height of the dielectric and plasmonic waveguides should match that of the silicon layer (i.e., $250 \mathrm{~nm}$ ). First, the dielectric waveguides are defined followed by depositing metal in the required areas. After that, the AGC and MDM waveguides are defined using the FIB. A platinum layer of a thickness of 7 to $10 \mathrm{~nm}$ is deposited on top of the metal layer before defining the AGC and MDM waveguides. The platinum layer is deposited to assist in getting a rectangular shape slot waveguide (i.e., the top of the slot waveguide has the same width as that of its bottom). 


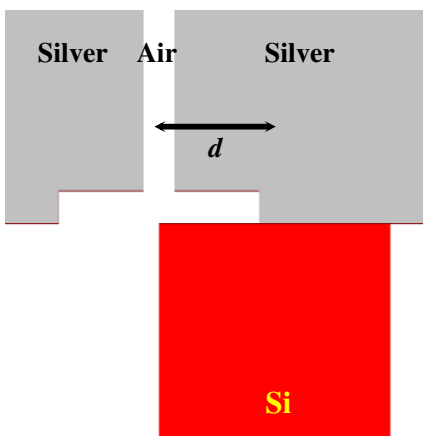

(a)

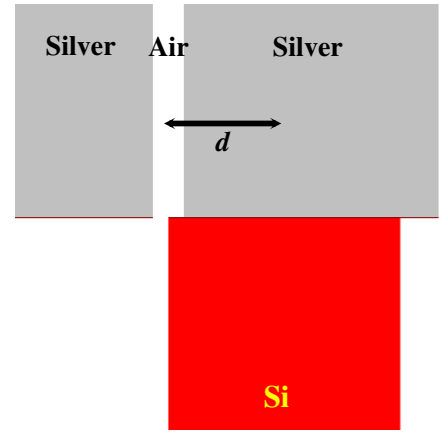

(d)

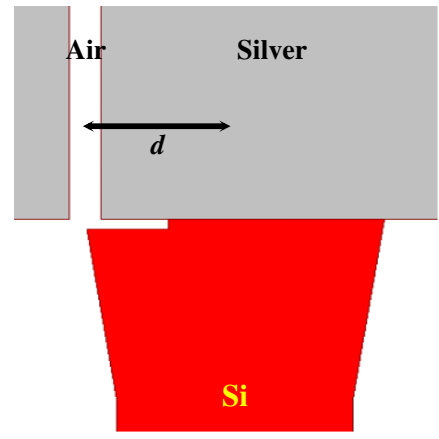

(f)

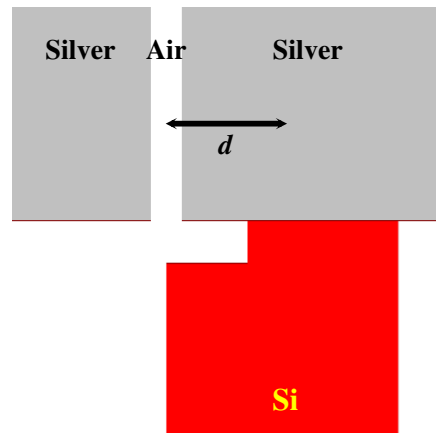

(b)

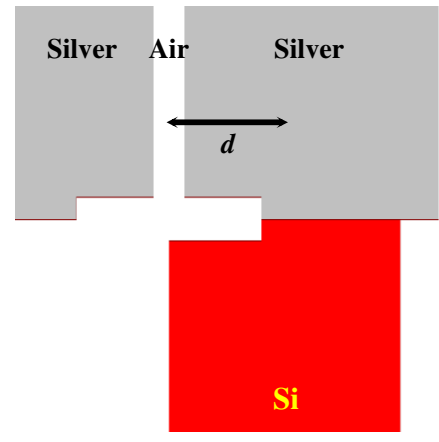

(c)

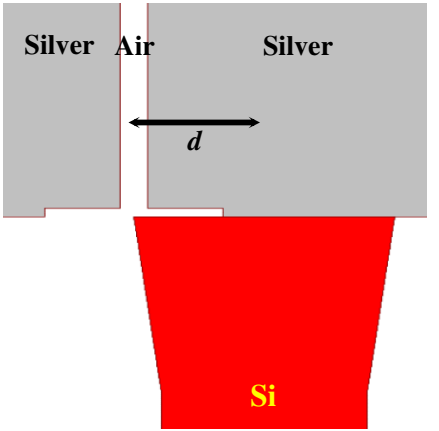

(e)

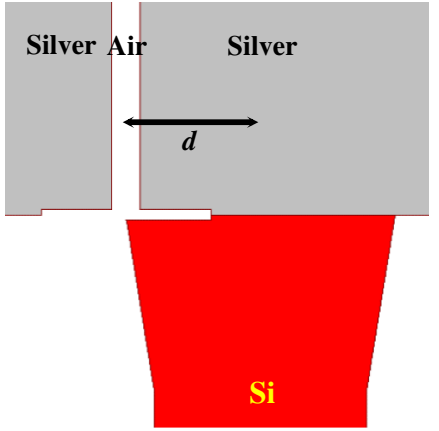

(g)

Fig. 5 Schematics of the misalignment distance, $d$, between the dielectric and MDM waveguides (with the AGC is kept connected to the MDM waveguide) for the following scenarios: (a) AGC \#1 without Si width expansion, (b) AGC \#2 without Si width expansion, (c) AGC \#3 without Si width expansion, (d) butt coupling, (e) AGC \#1 with Si width expansion, (f) AGC \#2 with Si width expansion, and (g) AGC \#3 with Si width expansion.

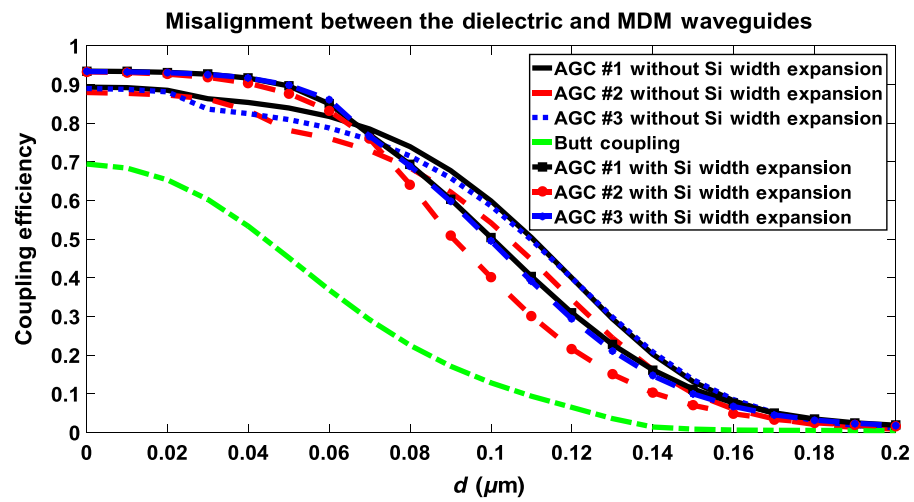

Fig. 6 CE as a function of the misalignment distance, $d$, for all schematics shown in Fig. 5. 


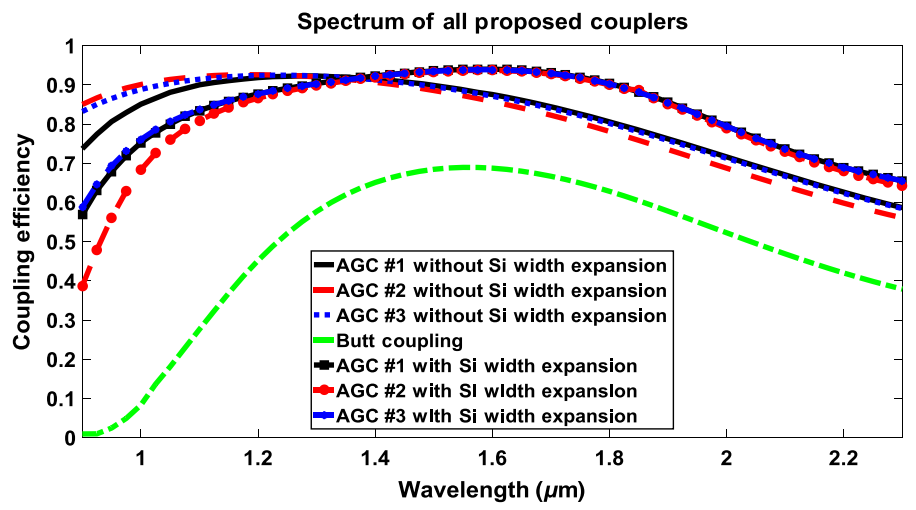

Fig. 7 Spectrum of all the proposed couplers that are shown in Figs. 1 and 3.

\section{Conclusions}

We proposed and optimized highly efficient couplers between a 300-nm-wide Si waveguide and 40-nm-wide silver-air-silver plasmonic waveguide. The proposed three types of AGCs resulted in a CE above $88 \%$ compared to $68 \%$ without using the AGCs (i.e., butt coupling). Further increase of the CE (above 93\%) was possible by increasing the width of the dielectric waveguide before it was connected to our proposed couplers. Moreover, the spectrum of all the proposed AGCs operates at a broad frequency range and provides large fabrication tolerance when aligning the dielectric and plasmonic MDM waveguides.

\section{References}

1. R. Zia et al., "Geometries and materials for subwavelength surface plasmon modes," J. Opt. Soc. Am. A 21(12), 2442-2446 (2004).

2. E. N. Economou, "Surface plasmons in thin films," Phys. Rev. 182(2), 539-554 (1969).

3. R. A. Wahsheh, Z. Lu, and M. A. G. Abushagur, "Nanoplasmonic couplers and splitters," Opt. Express 17(21), 19033-19040 (2009).

4. R. A. Wahsheh, Z. Lu, and M. A. G. Abushagur, "Nanoplasmonic directional couplers and Mach-Zehnder interferometers," Opt. Commun. 282(23), 4622-4626 (2009).

5. B. Wang and G. P. Wang, "Plasmon Bragg reflectors and nanocavities on flat metallic surfaces," Appl. Phys. Lett. 87(1), 013107 (2005).

6. H. Lu et al., "Enhancement of transmission efficiency of nanoplasmonic wavelength demultiplexer based on channel drop filters and reflection nanocavities," Opt. Express 19(14), 12885-12890 (2011).

7. K. Wen et al., "A four-port plasmonic quasi-circulator based on metal-insulator-metal waveguides," Opt. Express 20(27), 28025-28032 (2012).

8. Y. Gong, X. Liu, and L. Wang, "High-channel-count plasmonic filter with the metalinsulator-metal Fibonacci-sequence gratings," Opt. Lett. 35(3), 285-287 (2010).

9. G. Wang et al., "Numerical investigation of an all-optical switch in a graded nonlinear plasmonic grating," Nanotechnology 23(44), 444009 (2012).

10. P. Ginzburg, D. Arbel, and M. Orenstein, "Gap plasmon polariton structure for very efficient microscale-to-nanoscale interfacing," Opt. Lett. 31(22), 3288-3290 (2006).

11. S. H. Badri and M. M. Gilarlue, "Coupling between silicon waveguide and metal-dielectricmetal plasmonic waveguide with lens-funnel structure," Plasmonics 15, 821-827 (2020).

12. R. A. Wahsheh and M. A. G. Abushagur, "Experimental and theoretical investigations of an air-slot coupler between dielectric and plasmonic waveguides," Opt. Express 24(8), 8237-8242 (2016).

13. R. Yang et al., "Efficient light coupling between dielectric slot waveguide and plasmonic slot waveguide," Opt. Lett. 35(5), 649-651 (2010).

14. G. Veronis and S. Fan, "Theoretical investigation of compact couplers between dielectric slab waveguides and two-dimensional metal-dielectric-metal plasmonic waveguides," Opt. Express 15(3), 1211-1221 (2007). 
15. C. T. Chen et al., "High efficiency silicon strip waveguide to plasmonic slot waveguide mode converter," Proc. SPIE 9368, 936809 (2015).

16. V. Patel, P. Sharma, and V. D. Kumar, "Efficient coupling from dielectric to hybrid plasmonic waveguide using curved taper," IEEE Photonics Techol. Lett. 31(4), 323-326 (2019).

17. R. A. Wahsheh, Z. Lu, and M. A. G. Abushagur, "Ultra-compact integrated nanoplasmonic air-gap coupler," in Front. Opt., OSA, Paper FTh4E.5 (2014).

18. Z. Han, A. Y. Elezzabi, and V. Van, "Experimental realization of subwavelength plasmonic slot waveguides on a silicon platform," Opt. Lett. 35(4), 502-504 (2010).

19. L. Chen, J. Shakya, and M. Lipson, "Subwavelength confinement in an integrated metal slot waveguide on silicon," Opt. Lett. 31(14), 2133-2135 (2006).

Rami A. Wahsheh is an assistant professor at Princess Sumaya University for Technology, in Amman, Jordan. He received a bachelor's degree in electrical engineering in 1996 from Mutah University in Karak, Jordan, a master's degree in electrical engineering in 2002 from the University of Alabama in Huntsville, Huntsville, Alabama, USA, and a PhD in microsystems engineering from Rochester Institute of Technology in Rochester, New York, USA. His research work resulted in more than 20 publications. His research interests include optical devices in systems including photonic crystals, nanoplasmonics, and optical couplers. He is a member of OSA. 